This item was submitted to Loughborough's Research Repository by the author.

Items in Figshare are protected by copyright, with all rights reserved, unless otherwise indicated.

\title{
Working memory and number line representations in single-digit addition: approximate versus exact, nonsymbolic versus symbolic
}

PLEASE CITE THE PUBLISHED VERSION

http://dx.doi.org/10.1080/17470218.2014.977303

\section{PUBLISHER}

Taylor \& Francis (@ 2014 The Experimental Psychology Society)

\section{VERSION}

AM (Accepted Manuscript)

\section{PUBLISHER STATEMENT}

This work is made available according to the conditions of the Creative Commons Attribution-NonCommercialNoDerivatives 4.0 International (CC BY-NC-ND 4.0) licence. Full details of this licence are available at: https://creativecommons.org/licenses/by-nc-nd/4.0/

\section{LICENCE}

CC BY-NC-ND 4.0

\section{REPOSITORY RECORD}

Xenidou-Dervou, Iro, Menno van der Schoot, and Ernest C.D.M. van Lieshout. 2019. "Working Memory and Number Line Representations in Single-digit Addition: Approximate Versus Exact, Nonsymbolic Versus Symbolic". figshare. https://hdl.handle.net/2134/20088. 
Running head: EARLY MENTAL ADDITION

Working Memory and Number Line Representations in Single-digit Addition: Approximate versus Exact, Nonsymbolic versus Symbolic

Iro Xenidou-Dervou ${ }^{*}$, Menno van der Schoot, \& Ernest C. D. M. van Lieshout Department of Educational Neuroscience and LEARN! research institute for learning and education. Faculty of Psychology and Education, VU University Amsterdam, the Netherlands.

\section{Acknowledgement}

This work was supported by the NWO (National Dutch Organization for Scientific Research) under Grant number PROO 41107111.

*Corresponding author at:

Dept. of Educational Neuroscience and LEARN! research institute for learning and education, Faculty of Psychology and Education, VU University Amsterdam, Post address: Van der Boechorststraat 1, 1081 BT, Amsterdam, the Netherlands. Tel.: +31 (0)20 5989413 ; E-mail address: I.Xenidou-Dervou@,vu.nl (I. Xenidou-Dervou). 


\begin{abstract}
How do kindergarteners solve different single-digit addition problem formats? We administered problems that differed solely on the basis of two dimensions: response type (approximate or exact), and stimulus type (nonsymbolic, i.e. dots, or symbolic, i.e. Arabic numbers). We examined how performance differs across these dimensions, and which cognitive mechanism (mental model, transcoding, or phonological storage) underlies performance in each problem format with respect to working memory (WM) resources and mental number line representations. As expected, nonsymbolic problem formats were easier than symbolic ones. The visuospatial sketchpad was the primary predictor of nonsymbolic addition. Symbolic problem formats were harder because they either required the storage and manipulation of quantitative symbols phonologically or taxed more WM resources compared to their nonsymbolic counterparts. In symbolic addition, WM and mental number line results showed that when an approximate response was needed, children transcoded the information to the nonsymbolic code. When an exact response was needed, however, they phonologically stored numerical information in the symbolic code. Lastly, we found that more accurate symbolic mental number line representations were related to better performance in exact addition problem formats, not the approximate ones. This study extends our understanding of the cognitive processes underlying children's simple addition skills.
\end{abstract}

Keywords: Numerical Cognition, Kindergarten Children, Mental Model, Simple Arithmetic, Nonsymbolic and Symbolic addition. 
Working Memory and Number Line Representations in Single-digit Addition: Approximate versus Exact, Nonsymbolic versus Symbolic

The mastery of simple arithmetic is a prerequisite for the further development of mathematical competencies (Geary, 2011; Hamann \& Ashcraft, 1985; Price, Mazzocco, \& Ansari, 2013). A substantial body of research has demonstrated that early childhood nonsymbolic and symbolic numerosity processing skills, even before school entry, are important for later cognitive and educational development (for reviews see De Smedt, Noël, Gilmore, \& Ansari, 2013; Feigenson, Dehaene \& Spelke, 2004; Piazza, 2010). To enhance early numeracy skills performance, research must first identify how performance differs across the various problem formats and their underlying cognitive mechanisms. Kindergarten age is of special interest, as it is the preparatory stage before primary school entry. At this stage, mathematics instruction primarily focuses on teaching children how to solve simple addition problems with single-digits.

In the literature, one identifies several different addition problem formats. Despite the prominent roles that are being attributed to small numerosity nonsymbolic or symbolic processing skills (see De Smedt et al., 2013), very little is known about their underlying mechanisms. Addition in the form of " $\mathrm{a}+\mathrm{b}=\mathrm{c}$ " asks for an exact response and typically entails symbolic stimuli (i.e., Arabic numbers). This skill may take years to master (Hamann \& Ashcraft, 1985). Children, however, can already perform better in such addition problems from the preschool age, if instead of symbolic stimuli they entail nonsymbolic stimuli, e.g. objects like chips (Rasmussen \& Bisanz, 2005). Furthermore, developments in the field of numerical cognition have highlighted the importance of children's early abilities to add and compare quantities in problems that ask for an approximate response (e.g. "a + b" vs. "c", “which is more?") and entail nonsymbolic (Barth, La Mont, Lipton, \& Spelke, 2005; De 
Smedt, Verschaffel, \& Ghesquière, 2009; Gilmore, McCarthy, \& Spelke, 2010; Holloway \& Ansari, 2009; Mazzocco, Feigenson, \& Halberda, 2011; Xenidou-Dervou, van Lieshout, \& van der Schoot, 2014), or symbolic stimuli (Gilmore, McCarthy, \& Spelke, 2007; XenidouDervou, De Smedt, van der Schoot, \& van Lieshout, 2013).

Approximate and exact, nonsymbolic and symbolic mental representation skills are assumed to comprise the core systems that underlie our ability to process and manipulate numbers (Feigenson, Dehaene, \& Spelke, 2004; Piazza, 2010). However, very little is known about how kindergarteners' performance differs across the different problem formats.

Existing measures in the literature differ on the basis of multiple design characteristics. Also, little focus has been placed on these abilities' underlying cognitive mechanisms at the early stages of development. We developed and studied measures that permitted for the first time, to the best of our knowledge, the comparison of different single-digit addition problem formats on the basis of two important dimensions: 1) response type (approximate or exact), and 2) stimulus type (nonsymbolic or symbolic). Our aim was twofold: Firstly, to examine how kindergarteners' performance differs across the two dimensions. Secondly, to uncover the commonalities and differences of these abilities' cognitive profiles. On the basis of the mental model for simple arithmetic (Huttenlocher, Jordan, \& Levine, 1994; Rasmussen \& Bisanz, 2005), we examined which working memory (WM) component and which mental number line representation form relates to performance in these different addition problem formats.

Performance across the different single-digit addition problem formats.

Some problem formats are easier than others (Caviola, Mammarella, Cornoldi, \& Lucangeli, 2012; Kalaman \& Lefevre, 2007; Rasmussen \& Bisanz, 2005). Caviola et al., (2012), though, did not find this to be the case for the approximate versus exact problem 
format dimension. In their study, school-aged children (Grades 3 and 4) found the approximate and exact addition problems equally difficult. Notably, however, Caviola et al.'s (2012) experiments addressed complex (multidigit) addition skills. Research so far has not examined whether the same stands for simpler arithmetic, i.e. single-digit addition, in the case of novice arithmeticians, who may employ different solution strategies. Additionally, Caviola et al's (2012) study only addressed symbolic arithmetic. In contrast, Rasmussen and Bisanz (2005)'s work revealed the importance of the nonsymbolic versus symbolic ${ }^{1}$ dimension in single-digit mental arithmetic problems. The authors showed that novice learners perform better in nonsymbolic problems compared to symbolic ones. We presume that nonsymbolic problems are easier because nonsymbolic skills are evident already from infancy (Xu \& Spelke, 2000) and have been shown to require little or no previous instruction even with large numerosities (Barth et al., 2006; Gilmore et al., 2010; Xenidou-Dervou, et al., 2013, 2014). Furthermore, nonsymbolic approximate arithmetic skills are often thought to comprise the foundation for learning symbolic arithmetic (Gilmore et al., 2007; Mundy \& Gilmore, 2009; Xenidou-Dervou, et al., 2013). We, therefore, hypothesized that nonsymbolic single-digit addition problem formats would be easier than symbolic ones for kindergarten children.

But why is it that some problems are easier than others? The answer comes from research, which has demonstrated that different problem formats employ different cognitive mechanisms (Caviola et al., 2012; Kalaman \& Lefevre, 2007; Rasmussen \& Bisanz, 2005). The following sections describe the theoretical framework regarding two types of cognitive predictors of mental arithmetic, which comprised the focus of the present study; namely, WM and mental number line estimations. Their role in mental arithmetic is apprehended via the theoretical framework of the "mental model for simple arithmetic" (Huttenlocher et al., 1994; Rasmussen \& Bisanz, 2005). 


\section{The mental model in WM for single-digit addition.}

The mental model assumes that performance variations across different simple arithmetic problem formats occur because different presentation formats place different demands on one's WM (Huttenlocher et al., 1994; Rasmussen \& Bisanz, 2005). This is evidenced by the differential role of WM's components (Rasmussen \& Bisanz, 2005). Before we continue describing the mental model, however, a short description of WM and its subcomponents is rendered necessary.

WM refers to the domain general cognitive capacity that is responsible for the shortterm storage and manipulation of a limited amount of information; a necessary process for mental arithmetic (for reviews see DeStefano \& LeFevre, 2004; Raghubar, Barnes, \& Hecht, 2010). In the influential theoretical account of WM by Baddeley and Hitch (1974; see Baddeley, 2012), WM is conceived as a multicomponent construct. It comprises the Phonological Loop (PL), which is responsible for the storage of phonological information, the Visuospatial Sketchpad (VSSP), which retains visuo-spatial information, and the Central Executive (CE), which monitors, controls and regulates the processes of the other two systems. All three components of WM have been demonstrated to be essential for mental arithmetic processing although their roles vary according to the cognitive demands of a given mathematical problem format (Caviola et al., 2012; DeStefano \& LeFevre, 2004; Kalaman \& Lefevre, 2007; Simmons, Willis, \& Adams, 2012; Friso-van den Bos, van der Ven, Kroesbergen, \& van Luit, 2013) and the children's developmental stage (McKenzie, Bull, \& Gray, 2003; Passolunghi, Mammarella, \& Altoè, 2008; Rasmussen \& Bisanz, 2005).

According to the mental model, before the age of six, children perform better in nonsymbolic problems than in symbolic ones because they use a readily accessible mental model for representing the nonsymbolic quantities in their VSSP, e.g. by retaining a representation of each nonsymbolic item in their WM. This was evidenced by Rasmussen and 
Bisanz (2005), who showed that the VSSP was the best and only unique predictor of preschoolers' nonsymbolic exact addition performance. Symbolic problems, on the other hand, are harder because they require the manipulation of quantitative symbols, which are not so readily accessible for young novice learners. Rasmussen and Bisanz’s (2005) findings reveal two alternative cognitive processes that could be taking place when young learners solve a symbolic math problem: a) If a problem format is familiar and can be solved by means of a counting strategy, then children can store symbolic information phonologically and thus use a phonological approach to solve the problem, without needing to resort to a mental model. This process would demand PL resources since it regards the storage of phonological information. In agreement with this assumption, the authors found Grade 1 children's symbolic exact addition skills to be uniquely predicted by PL measures. b) If a problem cannot be solved via a formal math strategy like counting, i.e. if the children have no previous experience or relevant knowledge to a specific problem format, then they may attempt to transcode the symbolic information to the nonsymbolic code. This way they can represent them in their readily accessible mental model (VSSP). This transcoding process inherently calls for the manipulation of information in one's WM, which necessitates CE resources. Accordingly, symbolic exact addition performance at the beginning of the kindergarten year was predicted best by a CE measure in Rasmussen and Bisanz's (2005) study. In the present study, we tested kindergarteners at the end of their school year. We, therefore, expected that they would be familiar with the symbolic exact addition format and that the PL would be the best WM predictor, reflecting a phonological approach.

This theoretical framework provides a basis for understanding how young children solve nonsymbolic and symbolic exact addition problems. But do the mental model implications also extend to approximate problems? We expected that the difference in response type would not alter the result: the VSSP would be the primary predictor also for 
nonsymbolic approximate problems, as kindergarteners would represent the nonsymbolic quantities in their readily accessible mental model. Symbolic approximate addition, however, is not a problem format that they encounter within their kindergarten education, at least in the Netherlands. We, therefore, expected that the CE would best predict performance in this problem format, reflecting the aforementioned transcoding process.

The mental model, as described thus far, introduces clear predictions with respect to the role of the domain-general ability of WM and its' components in simple arithmetic. However, even though Rasmussen and Bisanz (2005) make reference to the way numerical information can be mentally (trans)coded from the symbolic to the nonsymbolic code, they did not test domain-specific coding, i.e. how math-specific information is mentally coded. The present study aimed to examine the mental model's assumptions with respect to WM's components and extend it by examining also domain-specific coding, namely nonsymbolic and symbolic mental number line representations and the role they play in conjunction with WM processing in solving different addition problem formats.

\section{The role of mental number line estimations.}

One of our aims was to explore what happens beyond the storage (i.e., WM) of numerical information. In which form is the numerical information mentally represented in the different addition problem formats? It is generally perceived that people mentally represent numerosities on a mental number line, an equivalent to a mental ruler (e.g., Aiello et al., 2012; Dehaene, Bossini, \& Giraux, 1993). Tasks assessing number line estimation typically ask participants to place a symbolic (i.e., Arabic) or a nonsymbolic (e.g., objects) numerosity on an empty number line that ranges, for example, from 0 to 10 and 0 to 100 (Booth \& Siegler, 2006; Sasanguie, De Smedt, Defever, \& Reynvoet., 2011; Siegler \& Booth, 2004). To the best of our knowledge, the question of whether different mental number line 
representations relate to performance in different addition problem formats has not been previously addressed. Below we report previous findings that broadly relate mental number line representations to approximate and general math achievement.

In approximate tasks with single-digits, the role of the mental number line is assumed to be evidenced via the so-called distance effect: the smaller the distance between two quantities is, the harder it is to compare them. This effect has been consistently demonstrated in both nonsymbolic and symbolic approximate tasks (e.g. De Smedt, Verschaffel, \& Ghesquière, 2009; Holloway \& Ansari, 2008, 2009; Sasanguie, et al., 2011). Even though performance on approximate magnitude measures has received a lot of research attention with respect to its role in children's mathematical achievement, very little is known so far about the underlying cognitive mechanisms of nonsymbolic and symbolic approximate number processing (De Smedt, Noël, Gilmore, \& Ansari, 2013; Gilmore, Attridge, De Smedt, \& Inglis, in press; Gilmore, Attridge, \& Inglis, 2011). In Sasanguie et al.'s (2011) study, the single-digit approximate measures did not correlate with their number line measures. Contrary to the rest of their tasks, however, the nonsymbolic number line task was a paper and pencil version. Also, the reliance of children's approximate judgments on the physical features of the nonsymbolic stimuli was not systematically controlled for in relation to the interval comparison quantity (i.e., target quantity vs the 10 or 100 nonsymbolic stimulus interval). For the present study, we developed computerized number line measures, in order to explore the specific relationship between mental number line representations and the four addition problem formats. For the first time, continuous quantity features of the nonsymbolic number line task were controlled for with the same methodology as that used in nonsymbolic approximate addition tasks (Barth et al., 2006; Gilmore et al., 2010; Xenidou-Dervou, et al., 2014). 
The relationship between mental number line representations and exact arithmetic is yet unclear. Mental number line representation skills have been associated with the development of children's general mathematics achievement (Booth \& Siegler, 2006; Sasanguie et al., 2011; Siegler \& Booth, 2004) but not exact arithmetic per se.

Based on the theoretical background presented so far, we generally hypothesized that mental number line representations would be also associated with approximate and exact mental arithmetic. As mentioned earlier, according to the mental model, in nonsymbolic approximate and exact addition each nonsymbolic element is assumed to be mentally represented as such. We, therefore, expected nonsymbolic mental number line representation to predict performance in the nonsymbolic problem formats. The case of symbolic addition is less straightforward. On the one hand, in symbolic exact addition, it is assumed that children store the symbolic information phonologically; so symbolic number line representation should be expected as a predictor. On the other hand, in symbolic approximate addition, we hypothesized that a transoding process would take place and thus nonsymbolic number line representation would best predict performance.

To summarize, the present study examined kindergarteners' performance in nonsymbolic and symbolic, approximate and exact single-digit addition problem formats. Our aim was two-fold: Firstly, to identify which problem formats are easier for children of this age. Secondly, to examine how the elements of the different problem formats are stored, manipulated and mentally represented. In other words, we scoped to uncover the cognitive correlates underlying performance in each of these problem formats and identify their underlying cognitive mechanisms (i.e., mental model, transcoding, phonological storage). In general, we hypothesized that nonsymbolic problem formats would be easier than symbolic ones due to differences in their underlying cognitive mechanisms; the first would rely primarily on the children's readily accessible mental model (Rasmussen \& Bisanz, 2005). 
Furthermore, we expected that the relationship between single-digit addition problem formats, WM and number line representations would depend on the characteristics of the addition problem format, namely: 1. Response type (approximate and exact), and 2. Stimulus type (nonsymbolic and symbolic). Table 1 summarizes our hypotheses for each single-digit addition problem format.

(Table 1 about here)

\section{Method}

\section{Participants}

We tested children from four urban kindergartens in the Netherlands. From the original sample four participants were removed from the analyses because they were identified as outliers (i.e., scored 3 standard deviations above/below the group mean in: symbolic approximate addition $[n=1]$, digit recall forwards $[n=1]$, digit recall backwards $[n$ $=1]$, or symbolic number line $0-100[n=1])$. The resulting sample consisted of 103 children (51 girls) $M_{\text {age }}=5.98, S D=0.40$. Passive consent was acquired from all participants' legal guardians. All children completed testing.

\section{Procedure}

Trained experimenters tested each child individually in a quiet setting within the school facilities. The tasks were introduced as games and were administered in three sessions, each lasting approximately thirty minutes. Between each testing session there was a time span of minimum one day and maximum two weeks. After each session participants received small tokens (e.g. stickers) to sustain their motivation and interest. 


\section{Materials}

Tasks used in this study were programmed in E-Prime, version 1.2 (Psychological Software Tools, Pittsburgh, PA, USA) and presented in HP Probook 6550b laptops.

Single-digit addition problem formats. Four addition tasks were used that differed only on the basis of type of stimulus and response. They all entailed 3 practice and 24 testing trials, the latter were presented in a random sequence (see Appendix Table A1). Instructions and feedback were provided only during practice. The addition tasks were adapted versions of the approximate addition tasks used in Xenidou-Dervou, et al. $(2013,2014)$. Every trial in these tasks entailed the images of a girl on the left side of the screen (Sarah) and a boy on the right side (Peter). Figure 1 depicts example trials from each of the four addition problem formats. Numerosities in these tasks ranged from 1 up to 9 as in well-known magnitude comparison tasks (e.g. Holloway \& Ansari, 2009) and simple arithmetic exact addition tasks (e.g. Rasmussen \& Bisanz, 2005). The numerical distance between the stimuli ranged from 1 up to 3 with 8 trials per distance block (see Appendix).

(Figure 1 about here)

Nonsymbolic approximate addition. In the nonsymbolic condition children saw (a) a set of blue dots appearing on the screen, (b) these were then covered up by a grey box, (c) then another set of blue dots fell inside the box, lastly (d) a set of red dots appeared on the right upper side of the screen and fell down (Figure 1). Each animated event lasted 1300ms and the interval between each step was $1200 \mathrm{~ms}$. Participants were asked to estimate "who got more dots, Sarah or Peter?" and to respond as correctly and as fast as possible. From the moment the red dots appeared on the screen, they had a maximum of $7000 \mathrm{~ms}$ to respond. To 
avoid responses being reliant on non-numerical characteristics, we used dot-stimuli where total dot surface area, total dot contour length and density were controlled for (see Appendix; Barth et al., 2006; Gilmore et al., 2010; Xenidou-Dervou et al., 2014). Dot size varied across the blue and red arrays: 10.5 or $3.5 \mathrm{~mm}$ diameter. All dot-stimuli were developed with MATLAB 7.5 R2007b.

Symbolic approximate addition. The symbolic condition was identical to the nonsymbolic with the sole difference of the stimuli presented. The children were told that Sarah and Peter would now play a game with numbers. First, Sarah would get blue boxes with a number of stickers in them and then Peter got a red box with a number of stickers in it. These boxes were labeled with Arabic numbers. Children were asked to estimate "who got more stickers, Sarah or Peter?" In both tasks the children responded by pressing the blue or the red response box situated in front of them.

Nonsymbolic exact addition. As depicted in Figure 1, the first two steps in the exact tasks were identical to the previously described approximate tasks. The sole difference of this nonsymbolic task with the approximate one laid in the response procedure. In the nonsymbolic condition children were asked to reproduce the "Exact amount of dots Sarah got". They could do this by pressing the "+" or "-” response buttons situated in front of them in order to create dots on the screen and a separate key when their response was ready. In order to encourage an exact response, no response time limit was set; only at the beginning of the task children had been instructed to respond as correctly and as fast as possible.

Symbolic exact addition. The symbolic condition was identical to the nonsymbolic one with the sole difference that now the children were instructed to show the "Exact number of stickers Sarah got" by pressing the correct number on the keyboard and a separate key when their response was ready. 
Predictors. We aimed to identify how digits or the numerosities they correspond to are mentally stored, manipulated and represented when kindergarteners solved the different single-digit addition problem formats in order to be able to make a distinction between the hypothesized underlying cognitive mechanisms (i.e., mental model, transcoding, phonological storage). In essence, we wanted our predictor tasks to differ with our dependent variables only on the key aspects of interest: storage, manipulation and representation. Therefore, we used WM and mental number line estimation tasks, which entailed only digits or nonsymbolic stimuli as in the addition problem formats.

WM. Kindergarteners' WM capacity was assessed with three widely used WM tasks (Alloway, Gathercole, Kirkwood, \& Elliott, 2008; Alloway, Gathercole, \& Pickering, 2006; Ang \& Lee, 2008; Rasmussen \& Bisanz, 2005).

Corsi Blocks. This task assessed VSSP WM (Ang \& Lee, 2008). A cross appeared and disappeared on one of nine randomly positioned squares on the screen. It entailed five span levels, with an extra cross appearing at each span. The children were given three practice trials: one for the first span (one cross), the second (two crosses) and the third (three crosses). Children were asked to recall the location and sequence of squares where the crosses appeared. They could respond by clicking on the corresponding squares. Each span entailed six trials. After four correct responses they automatically advanced to the next span, whereas three incorrect responses within one span terminated the task. The maximum score within one span was six. The outcome measure entailed the number of trials across the spans, where the child recalled correctly both the location and the presentation sequence of the crosses (for more information see Ang \& Lee, 2008). This task has demonstrated test-retest reliability .83 in children from 4.5 to 11.5 years (Alloway et al., 2006). 
Digit Span Forwards. This task measures children's PL capacity (Alloway, 2007). We used an adapted Dutch version. Children heard a sequence of recorded digits and were asked to repeat them correctly in the same order. They received two practice trials, one with one digit and one with two. The task entailed six spans: it started with one digit and continued up to the sequence of six digits. With four consecutive correct answers within one span, the child automatically advanced to the next span. Three incorrect responses within one span led to the termination of the task. The maximum score within one span was four. The outcome measure entailed the number of correctly repeated digits in the correct order. For children from 4.5 to 11.5 years, this task has demonstrated test-retest reliability .84 (Alloway et al., 2006).

Digit Span Backwards. This task assesses CE processing (Alloway, 2007; for the adapted version see Toll, Van der Ven, Kroesbergen, \& Van Luit, 2011). It was similar to the digit span forwards only this time the child had to recall the sequence of digits backwards. The task started with two digits. For children from 4.5 to 11.5 years, this task has demonstrated test-retest reliability .64 (Alloway et al., 2006).

Mental Number line Estimations. Two computerized tasks were developed: a nonsymbolic and a symbolic version (Figure 2). The tasks were based on the story of Pinocchio. A $25 \mathrm{~cm}$ empty number line that started from Pinocchio's nose was presented. Two intervals were administered in both conditions: $0-10$ and $0-100$ as in previous studies (e.g. Sasanguie et al., 2011). Children received four practice trials for each of the two intervals. The quantity to be positioned (target quantity), dots or number, was presented in a circle in the middle of the screen $(6.7 \mathrm{~cm}$ diameter $), 1.7 \mathrm{~cm}$ above the line. The intervals were indicated with two circles, $3 \mathrm{~cm}$ beneath the line on its far right and far left side. Zero was indicated with an empty circle. Children were asked to "pull" Pinocchio's nose using the mouse until it reached the point that they thought corresponded to the target quantity. By 
moving the mouse right or left, Pinocchio's nose grew larger or smaller on the line. The child had to click with the mouse to indicate the estimated position of the target quantity on the line. In the 0-10 interval, quantities 1 through 9 were presented. In the $0-100$ interval the following quantities were presented: $2,3,4,6,11,18,22,25,42,48,52,67,71,86$ (Sasanguie et al., 2011; Siegler \& Opfer, 2003).

Nonsymbolic stimuli were white circles with blue dots developed with the same method as in the nonsymbolic addition tasks (see Xenidou-Dervou et al., 2014). Dot size varied across the target and interval quantities: 12 or $2 \mathrm{mms}$ diameter (0-10 interval) and 3.5 or $1 \mathrm{~mm}$ diameter (0-100 interval). Therefore, contrary to previous nonsymbolic number line versions, in this task continuous quantity variables related to the target and the interval quantity were controlled for, namely: total dot surface area, total dot contour length and density. Symbolic stimuli were circles with blue Arabic digits made in Paint with 38 font size. For the outcome variables Percentages of Absolute Error (PAE) were computed (see Siegler \& Booth, 2004) based on the formula: |(Estimate - To Be Estimated Quantity)/Scale of Estimates|. Thus, if a child was asked to estimate the position of the number 67 on the $0-100$ interval and pulled the "nose" to the position corresponding to the number 90 on the line, then its PAE would correspond to $(90-67) / 100$ or $23 \%$.

It should be noted that recent research with kindergarteners has demonstrated that small numerosity nonsymbolic and symbolic processing skills are related to large-range (0-100) number line performance (Friso-van den Bos, Kroesbergen, \& van Luit, 2014). Therefore, we included small-range number lines (0-10) because the addition problems entailed small numerosities, but also large-range number lines (0-100). In essence, we were interested in how kindergarteners' general ability to map numbers onto a number line relate to their performance in simple addition problem formats, irrespective of specific strategies that may be employed (linear or loglinear) due to the range of the number line. Thus, in the analyses 
the children's mean PAE performance across all mental number line trials was used (Sasanguie et al., 2011), weighted on the basis of the number of trials within each range.

(Figure 2 about here)

\section{Results}

\section{Descriptive and preliminary analyses}

Table 2 presents descriptive statistics on all dependent measures and predictors. First, a series of analyses were conducted in order to verify that our approximate tasks indeed tapped the abilities in question. Participants performed above chance level (50\%) in both the nonsymbolic, $M=81.72 \%, t(102)=29.4, p<.001$, and the symbolic approximate addition task, $M=74.68 \%, t(102)=16.71, p<.001$. Also, the characteristic distance effects were found for performance in both approximate tasks: nonsymbolic, $F(2,204)=39.0, p<.001$, and symbolic $F(2,204)=14.52, p<.001$ (see Figure 3 ). This meant that the smaller the distance between the summed blue quantities and the red quantity was, the harder it was to compare them. Further analyses showed that children did not use systematic response strategies alternative to approximate addition (Appendix). Also, their responses in the nonsymbolic condition did not rely on the physical features of the dots (Appendix). Therefore, performance in these approximate addition tasks replicated previous characteristic findings (Barth et al., 2006; Gilmore et al., 2010; Xenidou-Dervou, et al., 2013; 2014).

(Table 2 about here) 


\section{Task comparisons}

All addition tasks had a maximum score of 24 . However, in the approximate tasks a score of 12 reflected chance level performance. These scores, therefore, were transformed on a percentage scale with $0 \%$ as chance level and $100 \%$ as highest score. When " $\mathrm{k}$ " was the number of trials (24) and "m" the child's performance on an approximate task then new scores were calculated with the formula: $100 \times\{[\mathrm{m}-(\mathrm{k} / 2)] /(\mathrm{k} / 2)\}$. Additionally, accuracy data on the exact tasks were re-scaled to percentages. A 2 × 2 repeated measures ANOVA was conducted with the two response types (approximate and exact) and the two types of stimuli (nonsymbolic and symbolic). Results showed a significant main effect for stimulus type, $F(1$, $102)=20.30, p<.001$, partial $\eta^{2}=.17$, on addition performance. No main effect for response type $(p=.16)$ or interaction effect $(p=.10)$ was found. As expected, kindergarteners' performance was significantly better in the nonsymbolic conditions $(M=59.93 \%)$ compared to the symbolic ones $(M=49.55 \%)$. Their performance did not differ, however, between the approximate $(M=56.39 \%)$ and exact tasks $(M=53.10 \%)$.

\section{Correlations}

Next, correlations were computed in order to determine which WM and number line variable correlated with each of the four different addition problem formats (Table 3). Age did not correlate with children's performance in any of the measures. Accuracy in all addition measures correlated significantly with the VSSP and PL measure. The CE only correlated with symbolic approximate and nonsymbolic exact addition. Furthermore, performance in all addition tasks correlated with both number line measures with the exception of the nonsymbolic approximate addition task, which did not correlate with either of the number line measures. Further analyses revealed possible sources of performance differentiation across the different addition problem formats. 
(Table 3 about here)

\section{Regression analyses}

We were interested in which WM and number line measure predicted performance in each of the four addition problem formats. We did not have specific hypotheses with respect to the order of importance of the predictors for all dependent variables. We, therefore, conducted four stepwise multiple linear regression analyses for each addition problem format with their respective predictors ${ }^{2}$. Table 4 shows the regression analyses' results and Table 5 depicts a summarized representation of these findings alongside the corresponding mental process proposed.

(Table 4 about here)

Nonsymbolic Approximate. Results showed that the VSSP, $t(102)=2.83, p=.006$, was the best and only unique predictor of nonsymbolic approximate addition. As evidenced in the correlation analyses, performance in this task did not correlate with either of the two mental number line estimation tasks. Thus, one may doubt whether the nonsymbolic approximate task used in this study actually tapped the desired ability. We found that children performed highly above chance level and replicated the characteristic distance effect. However, five trials in our nonsymbolic approximate task could have been subitized and not estimated approximately (see Table A1; trials 2, 5, 6, $12 \& 15$ ). Subitizing is a mental process, which reflects the accurate numeration of sets up to 3 or 4 (Revkin, Piazza, Izard, Cohen, \& Dehaene, 2008). In these trials, both the summed addends and the comparison quantity belonged in this small number-range category (1-4) and could have thus been solely subitized. We, therefore, re-ran the analyses having removed these trials. Results remained the same. 
Once again, nonsymbolic approximate addition did not correlate with either the nonsymbolic $(p=.117)$ or the symbolic number line estimation task $(p=.350)$.

Symbolic Approximate. As expected, nonsymbolic number line estimation, $t(102)=-$ 4.03, $p<.001$, the VSSP, $t(102)=2.91, p=.004$ and the CE, $t(102)=2.18, p=.032$, were demonstrated to be significant predictors of symbolic approximate addition. This pattern of results is consistent with the transcoding assumption (involvement of the $\mathrm{CE}$ ), which permits the quantities to be transcoded to the nonsymbolic code and thus be processed in ones mental model (VSSP).

Nonsymbolic Exact. Results showed that, for kindergarteners the VSSP was the best WM predictor (see beta values in Table 4) of nonsymbolic exact addition in the multiple regression model, $t(102)=5.89, p<.001$. Aside from the VSSP, though, the symbolic mental number line predictor, $t(102)=-3.73, p<.001$ and the CE, $t(102)=2.00, p=.049$ also explained significant variance. These results suggest an alternative transcoding process. Namely, that the nonsymbolic quantities mentally represented in the VSSP can be transcoded via the $\mathrm{CE}$ to the symbolic code (symbolic number line estimation).

Symbolic Exact. In the case of symbolic exact addition, as expected, children's performance in the symbolic mental number line representation task, $t(102)=-3.89, p<.001$, and the PL, $t(102)=2.97, p=.004$, were identified as the significant predictor variables. 


\section{Discussion}

The present study examined kindergarteners' performance in four addition problem formats that differed solely on the basis of response type (approximate or exact) and stimulus type (nonsymbolic or symbolic). Our aim was to examine how performance differs across these problem formats and to uncover their underlying cognitive mechanisms (i.e., mental model, transcoding, or phonological storage). Specifically, we sought for the commonalities and differences in these addition skills' cognitive profiles on the basis of the mental model for simple arithmetic (Huttenlocher et al., 1994; Rasmussen \& Bisanz, 2005). We expected that nonsymbolic problems would be easier compared to symbolic ones and that different combinations of WM components and mental number line representations would be related to performance in the different problem formats. These expectations were confirmed (Table 5).

We found that kindergarteners' performance did not differ on the basis of the response type of the addition problem (approximate or exact); both problem formats were equally difficult for them. Caviola et al., (2012) evidenced the same result for school-aged children when comparing their performance across symbolic approximate and exact tasks. Our study extends previous findings by showing that what makes a difference in the level of difficulty of an arithmetic problem format (either approximate or exact) is the type of stimuli used. As expected, problem formats with nonsymbolic stimuli were easier than the symbolic ones. Consistent with the assumptions put forward by Huttenlocher et al. (1994) and Rasmussen \& Bisanz (2005), these problems were easier because their performance seemed to primarily rely on a readily accessible cognitive system allowing them to be solved without needing a lot of previous instruction. This system necessitates the VSSP (Huttenlocher et al., 1994; Rasmussen \& Bisanz, 2005) and, accordingly, the VSSP was the primary predictor of both exact and approximate nonsymbolic addition. In the following section, we discuss our findings for each single-digit problem format. 
Our results demonstrated for the first time that the VSSP was the best and only WM predictor of single-digit nonsymbolic approximate addition. Contrary to large-numerosity nonsymbolic approximate addition, which necessitates the CE component of WM in order to process condensed whole arrays (Xenidou-Dervou, et al., 2014), single-digit nonsymbolic approximate addition was not related to CE processing. Thus, as expected, kindergarteners' performance in this task reflected the use of a mental model for representing quantities in their WM (Huttenlocher et al., 1994; Rasmussen \& Bisanz, 2005). Notably, performance in this problem format did not correlate with either of the two number line measures as in the case of Sasanguie et al.'s (2011) results.

The assumption that mental number line representation underlies approximate estimation has been derived from findings relating numerical distance with spatial distance (e.g. Dehaene et al., 1993). Barth and Paladino (2011), however, have suggested that mental number line estimation tasks may actually tap the ability to judge proportions. Our results suggest that single-digit nonsymbolic approximate addition is not related to the ability to estimate quantities or proportions on a mental number line. This finding may be attributed to the different nature of the nonsymbolic approximate addition task and the number line tasks: the first asks for an approximate estimation, whereas the latter ask for a more precise estimation of the target. We found that nonsymbolic approximate tasks with single-digits primarily reflect children's VSSP capacity. Nonsymbolic approximate magnitude measures with numerosities ranging from 1 up to 9 (as in our task) have been widely used within the literature but their underlying mechanisms had been so far largely unexplored. The most well known ones are the magnitude comparison tasks (e.g. Holloway \& Ansari, 2009; Nosworthy, Bugden, Archibald, Evans, \& Ansari, 2013; Sasanguie et al., 2011). Our findings suggest that VSSP WM capacity should be taken into account when examining the role of such small- 
numerosity approximate magnitude tasks in young children and raise the question of to what extent do such tasks assess numerical estimation beyond VSSP capacities.

In the case of symbolic approximate addition, as expected, our results showed that performance in this problem format was related with nonsymbolic mental number line representation, the CE and the VSSP. This pattern of results supported the assumption that at this young age in unfamiliar problem formats children transcode symbolic information to the nonsymbolic code in order to process them in their mental model (Rasmussen \& Bisanz, 2005). This way, the symbolic information can be held in memory in its original code (symbolic) while translating it via the $\mathrm{CE}$ into the nonsymbolic code (nonsymbolic mental number line representation), in order for it to be represented in the readily accessible system that requires the VSSP (Rasmussen \& Bisanz, 2005). Previous research had not addressed domain-specific coding, namely what happens beyond the storage of numerical information in ones' WM. These findings demonstrated for the first time the collective roles that mental number line representation and WM components play in symbolic approximate mental addition. Aside from the nonsymbolic number line estimation skills, the CE and the VSSP were rendered as significant predictors. Symbolic approximate addition, therefore, placed a significant amount of cognitive demands on the kindergarteners, making it a harder problem format than its nonsymbolic counterpart.

In the nonsymbolic exact addition problem format, besides the expected primary role of the VSSP, the CE and symbolic mental number line representation were unexpectedly also shown to be contributing predictors reflecting an alternate type of a transcoding mechanism. This is contradictory to Rasmussen and Bisanz's (2005) finding where the VSSP was the only predictor of nonsymbolic exact addition and our hypothesis that nonsymbolic number line representation would predict performance in this addition task. On the contrary, results suggested that the nonsymbolic information of the addition task may have been mentally 
transcoded to the symbolic form via the CE. A key difference between our study and that of Rasmussen and Bisanz's (2005), was the time-point within the school year when the kindergarteners were tested. In our study they were tested at the end of their school year, when they were already very familiar with symbolic exact addition problems of the form "a + $\mathrm{b}=\mathrm{c}$ ". It seems as if, at this developmental stage, the preferred representation mode for exact problem formats is the symbolic code, i.e., the solution of the addition problem being represented in its Arabic numeral form. Thus, we assume that some children instead of using solely their mental model to represent this nonsymbolic information (VSSP), they attempted to transcode it into the symbolic code via their $\mathrm{CE}$ in order to produce the appropriate exact response. In Table 5, one notices that in symbolic approximate addition the opposite transcoding process was reflected. Our findings, therefore, suggest that based on the response and stimulus type of a given problem format, children may use different transcoding processes in order to produce the appropriate responses. As this is the first indication of alternate transcoding processes taking place when solving different simple addition problems, this interpretation should be considered as tentative and future research should further examine the different transcoding processes that may take place when solving different math problem formats.

Symbolic exact addition, is a problem format with which kindergarteners are very familiar with, namely the “ $a+b=c$ ” form of math problems. Rasmussen and Bisanz's (2005) findings in exact symbolic addition had suggested that when a symbolic problem format is familiar, then the children could use a phonological approach to solve the problem. As expected, kindergarteners' PL and symbolic mental number line estimation skills predicted their symbolic exact addition performance. This suggested that they could solve this problem format by phonologically storing numerical information in the symbolic code. Thus, performance in this task was related to previous experience with symbolic arithmetic and not 
with the children's readily accessible mental model, constituting symbolic exact addition a harder problem format than its nonsymbolic version.

\section{General remarks}

The present study’s findings demonstrated that symbolic single-digit problem formats (approximate or exact) are harder than their nonsymbolic counterparts because they tend to either tax more WM resources or require the phonological storage and manipulation of quantitative symbols. Our findings extend the existing literature by: a) demonstrating the interrelationship of storage, manipulation and representation of numerosities when solving different types of simple addition problem formats, b) uncovering the different cognitive mechanisms - mental model, transcoding or phonological storage - which may take place while solving nonsymbolic or symbolic, approximate or exact simple addition problems.

One of the outstanding questions in numerical cognition relates to not only identifying predictors of arithmetic performance but also their interrelations and integrative roles (Xenidou-Dervou et al., 2013). The present study demonstrated how different combinations of WM predictors reflect different underlying cognitive mechanisms for the first time in conjunction with domain-specific coding, such as mental number line estimation skills. With respect to the role of number line representations, our findings provided proof for the mental model assumptions with respect to domain-specific coding, and highlighted for the first time their differential relation to solving different single-digit addition problem formats. We found that more accurate symbolic number line representations relate to better performance in exact addition problem formats, but not the approximate ones. In other words, the better kindergarteners could mentally represent symbolic notations, the better they could perform on math problems of the form " $a+b=c$ " with either nonsymbolic or symbolic stimuli. More accurate nonsymbolic number line representations, on the other hand, were related to 
performance in a problem format, which is not familiar to children, namely symbolic approximate addition.

In general, the literature so far, has focused on the differential predictive role of nonsymbolic and symbolic magnitude estimation abilities in children's general math achievement (De Smedt \& Gilmore, 2011; Holloway \& Ansari, 2009; Sasanguie et al., 2011; Xenidou-Dervou, et al., 2013). Despite the importance of this issue, though, it is also important to understand how these abilities affect performance in different arithmetic problem formats. Our findings comprise a stepping-stone towards this direction. We did not, however, address the different number line ranges as different predictors. This was done for statistical and psychometric reasons (e.g. would lead to too many predictors in total and too few trials within the $0-10$ range); moreover this issue was beyond the immediate scope of the study. Developmental research in this domain, though, should examine the role of the different mental number line ranges and the issue of linear versus logarithmic number line representation when addressing the prediction of different math problem formats, since evidence suggests that different number line ranges demonstrate differential developmental trajectories (Siegler \& Booth, 2004). There is also the question of whether number line tasks actually assess numerical magnitude processing (Barth \& Paladino, 2011). Especially, in light of the absence of a relation between nonsymbolic approximate addition and number line estimation, we suggest that future research addresses alternative questions of underlying number processing, such as magnitude comparison (e.g. Holloway \& Ansari, 2009).

Future research should also shed further light in the solution processes involved in the different single-digit problem formats. It would be interesting, for example, to examine the different strategies employed when solving nonsymbolic and symbolic single-digit problem formats and execute more rigorous experimental tests in order to examine the online role of the proposed underlying cognitive mechanisms. This can be done with dual-task designs, 
which can be applied in children as young as pre-school (Xenidou-Dervou et al., 2014). Furthermore, the relatively small amount of variance explained in certain regression models, indicates that further underlying skills should be examined in conjunction with WM and number line representation, such as subitizing, magnitude comparison or counting skills. Lastly, it would be interesting for future research to examine the role of WM in these problem formats also with math non-specific WM predictors, as well as use a CE measure that taps on the VSSP subcomponent of WM.

In summary, the present experiment's findings introduce new insights into understanding how children conduct simple arithmetic before they start primary school instruction. Especially from an educational design perspective, our findings demonstrate how specific variations amongst addition problem formats relate to different cognitive processes, which are important for the development of children's mathematical progress and achievement. 


\section{Footnotes}

${ }^{1}$ In their study, Rasmussen and Bisanz (2005) address nonverbal (i.e., chips) and verbal quantities (i.e. verbal formulation of the symbolic problem). In essence, though, what they called "nonverbal" quantities is the same as what we and other authors mean with "nonsymbolic" quantities.

${ }^{2}$ We also conducted two sets of reversed hierarchical multiple regressions for each problem format with the group of WM measures in one step and the group of number line predictors in the other. The measures within each group of predictors were entered stepwise. Results were similar; therefore, for clarity purposes only the single stepwise linear regression results are reported. 


\section{Acknowledgements}

The authors would like to thank all children, parents, teachers and schools that participated in this study. We would also like to thank Cor Stoof and Jarik den Hartog for their invaluable technical assistance in developing the tasks.

This work was supported by the NWO (National Dutch Organization for Scientific Research) under Grant number PROO 41107111. 
References

Alloway, T. P. (2007). Automated Working Memory Assessment (AWMA). London, UK: Pearson Assessment.

Aiello, M., Jacquin-Courtois, S., Merola, S., Ottaviani, T., Tomaiuolo, F., Bueti, D., ... Doricchi, F. (2012). No inherent left and right side in human "mental number line": evidence from right brain damage. Brain: A Journal of Neurology, 135, 2492-505. doi:10.1093/brain/aws114

Alloway, T., Gathercole, S. E., Kirkwood, H., \& Elliott, J. (2008). Evaluating the validity of the Automated Working Memory Assessment. Educational Psychology, 28, 725-734. doi:10.1080/01443410802243828

Alloway, T., P., Gathercole, S. E., \& Pickering, S. J. (2006). Verbal and visuospatial shortterm and working memory in children: are they separable? Child Development, 77, 1698-716. doi:10.1111/j.1467-8624.2006.00968.x

Ang, S. Y., \& Lee, K. (2008). Central executive involvement in children's spatial memory. Memory, 16, 918-33. doi:10.1080/09658210802365347

Baddeley, A. (2012). Working memory: theories, models, and controversies. Annual Review of Psychology, 63, 1-29. doi:10.1146/annurev-psych-120710-100422

Barth, H., La Mont, K., Lipton, J., Dehaene, S., Kanwisher, N., \& Spelke, E. (2006). Nonsymbolic arithmetic in adults and young children. Cognition, 98, 199-222. doi:10.1016/j.cognition.2004.09.011

Barth, H., La Mont, K., Lipton, J., \& Spelke, E. S. (2005). Abstract number and arithmetic in preschool children. Proceedings of the National Academy of Sciences of the United States of America, 102, 14116-21. doi:10.1073/pnas.0505512102

Barth, H., \& Paladino, A. M. (2011). The development of numerical estimation: evidence against a representational shift. Developmental Science, 14, 125-135. doi: $10.1111 / \mathrm{j} .1467-7687.2010 .00962 . x$

Caviola, S., Mammarella, I. C., Cornoldi, C., \& Lucangeli, D. (2012). The involvement of working memory in children's exact and approximate mental addition. Journal of Experimental Child Psychology, 112, 141-60. doi:10.1016/j.jecp.2012.02.005

De Smedt, B., \& Gilmore, C. K. (2011). Defective number module or impaired access? Numerical magnitude processing in first graders with mathematical difficulties. Journal of Experimental Child Psychology, 108, 278-92. doi:10.1016/j.jecp.2010.09.003

De Smedt, B., Noël, M.-P., Gilmore, C., \& Ansari, D. (2013). How do symbolic and nonsymbolic numerical magnitude processing relate to individual differences in children's mathematical skills? A review of evidence from brain and behavior. Trends in Neuroscience and Education, 1-8. doi:10.1016/j.tine.2013.06.001 
De Smedt, B., Verschaffel, L., \& Ghesquière, P. (2009). The predictive value of numerical magnitude comparison for individual differences in mathematics achievement. Journal of Experimental Child Psychology, 103, 469-79. doi:10.1016/j.jecp.2009.01.010

Dehaene, S., Bossini, S., \& Giraux, P. (1993). The mental representation of parity and number magnitude. Journal of Experimental Psychology: General, 122, 371-396.

DeStefano, D., \& LeFevre, J. (2004). The role of working memory in mental arithmetic. European Journal of Cognitive Psychology, 16(3), 353-386. doi:10.1080/09541440244000328

Friso-van den Bos, I., van der Ven, E., Kroesbergen, E. H., \& van Luit, J. E. H. (2013). Working memory and mathematics in primary school children: A meta-analysis. Educational Research Review, 10, 29-44. 10.1016/j.edurev.2013.05.003

Feigenson, L., Dehaene, S., \& Spelke, E. (2004). Core systems of number. Trends in Cognitive Sciences, 8, 307-14. doi:10.1016/j.tics.2004.05.002

Geary, D. C. (2011). Consequences, characteristics, and causes of mathematical learning disabilities and persistent low achievement in mathematics. Journal of Developmental and Behavioral Pediatrics, 32, 250-63. doi:10.1097/DBP.0b013e318209edef.

Gilmore, C., Attridge, N., \& Inglis, M. (2011). Measuring the approximate number system. The Quarterly Journal of Experimental Psychology, 64(11), 2099-2109.

Gilmore, C. K., McCarthy, S. E., \& Spelke, E. S. (2007). Symbolic arithmetic knowledge without instruction. Nature, 447, 589-91. doi:10.1038/nature05850

Gilmore, C. K., McCarthy, S. E., \& Spelke, E. S. (2010). Non-symbolic arithmetic abilities and mathematics achievement in the first year of formal schooling. Cognition, 115, 394406. doi:10.1016/j.cognition.2010.02.002

Hamann, M. S., \& Ashcraft, M. H. (1985). Simple and complex mental addition across development. Journal of Experimental Child Psychology, 40, 49-72. doi:10.1016/00220965(85)90065-7

Holloway, I. D., \& Ansari, D. (2009). Mapping numerical magnitudes onto symbols: the numerical distance effect and individual differences in children's mathematics achievement. Journal of Experimental Child Psychology, 103, 17-29. doi:10.1016/j.jecp.2008.04.001

Huttenlocher, J., Jordan, N. C., \& Levine, S. C. (1994). A mental model for early arithmetic. Journal of Experimental Psychology. General, 123, 284-96. Retrieved from http://www.ncbi.nlm.nih.gov/pubmed/7931093

Kalaman, D. a., \& Lefevre, J.-A. (2007). Working memory demands of exact and approximate addition. European Journal of Cognitive Psychology, 19, 187-212. doi:10.1080/09541440600713445 
Mazzocco, M. M. M., Feigenson, L., \& Halberda, J. (2011). Preschoolers' Precision of the Approximate Number System Predicts Later School Mathematics Performance, 6, 1-8. doi:10.1371/journal.pone.0023749

McKenzie, B., Bull, R., \& Gray, C. (2003). The effects of phonological and visual-spatial interference on children's arithmetical performance. Educational and Child Psychology, 20(3), 93-108.

Mundy, E., \& Gilmore, C. K. (2009). Children's mapping between symbolic and nonsymbolic representations of number. Journal of Experimental Child Psychology, 103, 490-502. doi:10.1016/j.jecp.2009.02.003

Noël, M.-P. (2009). Counting on working memory when learning to count and to add: a preschool study. Developmental Psychology, 45, 1630-43. doi:10.1037/a0016224

Nosworthy, N., Bugden, S., Archibald, L., Evans, B., \& Ansari, D. (2013). A Two-Minute Paper-and-Pencil Test of Symbolic and Nonsymbolic Numerical Magnitude Processing Explains Variability in Primary School Children's Arithmetic Competence. PLOS ONE, 8, e67918. doi: 10.1371/journal.pone.0067918

Opfer, J., \& Siegler, S. (2007). Representational change and children's numerical estimation. Cognitive Psychology, 55, 169-195.

Passolunghi, M. C., Mammarella, I. C., \& Altoè, G. (2008). Cognitive abilities as precursors of the early acquisition of mathematical skills during first through second grades.

Developmental Neuropsychology, 33, 229-250. doi: 10.1080/87565640801982320

Piazza, M. (2010). Neurocognitive start-up tools for symbolic number representations. Trends in Cognitive Sciences, 14, 542-51. doi:10.1016/j.tics.2010.09.008

Price, G. R., Mazzocco, M. M. M., \& Ansari, D. (2013). Why mental arithmetic counts: brain activation during single digit arithmetic predicts high school math scores. The Journal of Neuroscience, 33, 156-63. doi:10.1523/JNEUROSCI.2936-12.2013

Raghubar, K. P., Barnes, M. A., \& Hecht, S. A. (2010). Working memory and mathematics: A review of developmental, individual difference, and cognitive approaches. Learning and Individual Differences, 20, 110-122. 10.1016/j.lindif.2009.10.005

Rasmussen, C., \& Bisanz, J. (2005). Representation and working memory in early arithmetic. Journal of Experimental Child Psychology, 91, 137-157. doi:10.1016/j.jecp.2005.01.004

Revkin, S. K., Piazza, M., Izard, V., Cohen, L., \& Dehaene, S. (2008). Does subitizing reflect numerical estimation? Psychological science, 19, 607-14. doi:10.1111/j.14679280.2008.02130.x

Sasanguie, D., De Smedt, B., Defever, E., \& Reynvoet, B. (2011). Association between basic numerical abilities and mathematics achievement. The British Journal of Developmental Psychology, 30, 344-57. doi:10.1111/j.2044-835X.2011.02048.x 
Sekuler, R., \& Mierkiewicz, D. (1977). Children's Judgments of Numerical Inequality. Child Development, 48, 630-633. doi:10.2307/1128664

Siegler, R. S., \& Booth, J. L. (2004). Development of numerical estimation in young children. Child Development, 75(2), 428-44. doi:10.1111/j.1467-8624.2004.00684.x

Siegler, R. S., \& Opfer, J. E. (2003). The development of numerical estimation: evidence for multiple representations of numerical quantity. Psychological Science, 14, 237-43. Retrieved from http://www.ncbi.nlm.nih.gov/pubmed/12741747

Simmons, F. R., Willis, C., \& Adams, A.-M. (2012). Different components of working memory have different relationships with different mathematical skills. Journal of Experimental Child Psychology, 111, 139-55. doi:10.1016/j.jecp.2011.08.011

Toll, S. W. M., Van der Ven, S. H. G., Kroesbergen, E. H., \& Van Luit, J. E. H. (2011). Executive functions as predictors of math learning disabilities. Journal of Learning Disabilities, 44(6), 521-32. doi:10.1177/0022219410387302

Xenidou-Dervou, I., De Smedt, B., van der Schoot, M., \& van Lieshout, E. C. D. M. (2013). Individual differences in kindergarten math achievement: The integrative roles of approximation skills and working memory. Learning and Individual Differences, 28, 119-129. doi:10.1016/j.lindif.2013.09.012

Xenidou-Dervou, I., van Lieshout, E. C. D. M., \& van der Schoot, M. (2014). Working Memory in Nonsymbolic Approximate Arithmetic Processing: A Dual-Task Study With Preschoolers. Cognitive Science, 38, 101-127. doi:10.1111/cogs.12053

Xu, F., \& Spelke, E. S. (2000). Large number discrimination in 6-month-old infants. Cognition, 74, B1-B11. doi:10.1016/S0010-0277(99)00066-9 


\section{Appendix}

The present study's approximate addition tasks were designed based on well known approximate magnitude tasks. They entailed the presentation format of approximate addition tasks (Barth et al., 2006; Gilmore et al., 2007; Xenidou-Dervou, et al., 2013) and the numerosity range of magnitude comparison tasks (De Smedt, Verschaffel, \& Ghesquière, 2009; Holloway \& Ansari, 2009; Sekuler \& Mierkiewicz, 1977). This numerosity range, namely one up to nine, could be directly compared with corresponding single-digit exact addition tasks (Huttenlocher et al., 1994; Rasmussen \& Bisanz, 2005). Table A1 shows the 24 trials used across all addition problem formats. They were designed in such a manner that allowed post-hoc examination of possible strategies alternative to approximate addition (e.g., Xenidou-Dervou et al., 2014).

Since the numerosity range in our addition tasks was much smaller compared to other approximate tasks (Xenidou-Dervou et al., 2013, 2014)., we could by default control for less alternative systematic response preferences. In Table A1, under the "strategy response preferences column", two alternative strategies are shown: Strategy "Blue" controlled for children's possible tendency to press only the blue response button. Strategy "red" reflects the respective control for the red response button. A trial listed as 1 indicated that the strategy predicted the correct response in this trial, whereas - 1 signifies the prediction of an incorrect response. If children made use of one of the two strategies, then they would have performed at chance level (50\%). Results in Table A2, demonstrated that neither strategy was used in the nonsymbolic and the symbolic approximate addition problem. 
Furthermore, we examined whether children's responses were reliant on combined feature-variables of the dots in the nonsymbolic addition problem: namely, dot size, summed dot surface area, summed dot circumference, density and array total area (Barth et al., 2006; Gilmore et al., 2007; Xenidou-Dervou et al., 2013). As shown in Table A1 (continuous quantity column), half of the trials were presented in condition A, where dot size, summed dot surface area, total dot contour length and density were positively correlated with number. In condition B, these dot-feature characteristics were negatively correlated with number. Children performed above chance level in both conditions (Table A2) indicating that their responses were not reliant on continuous quantity variables.

(Table A2 about here) 

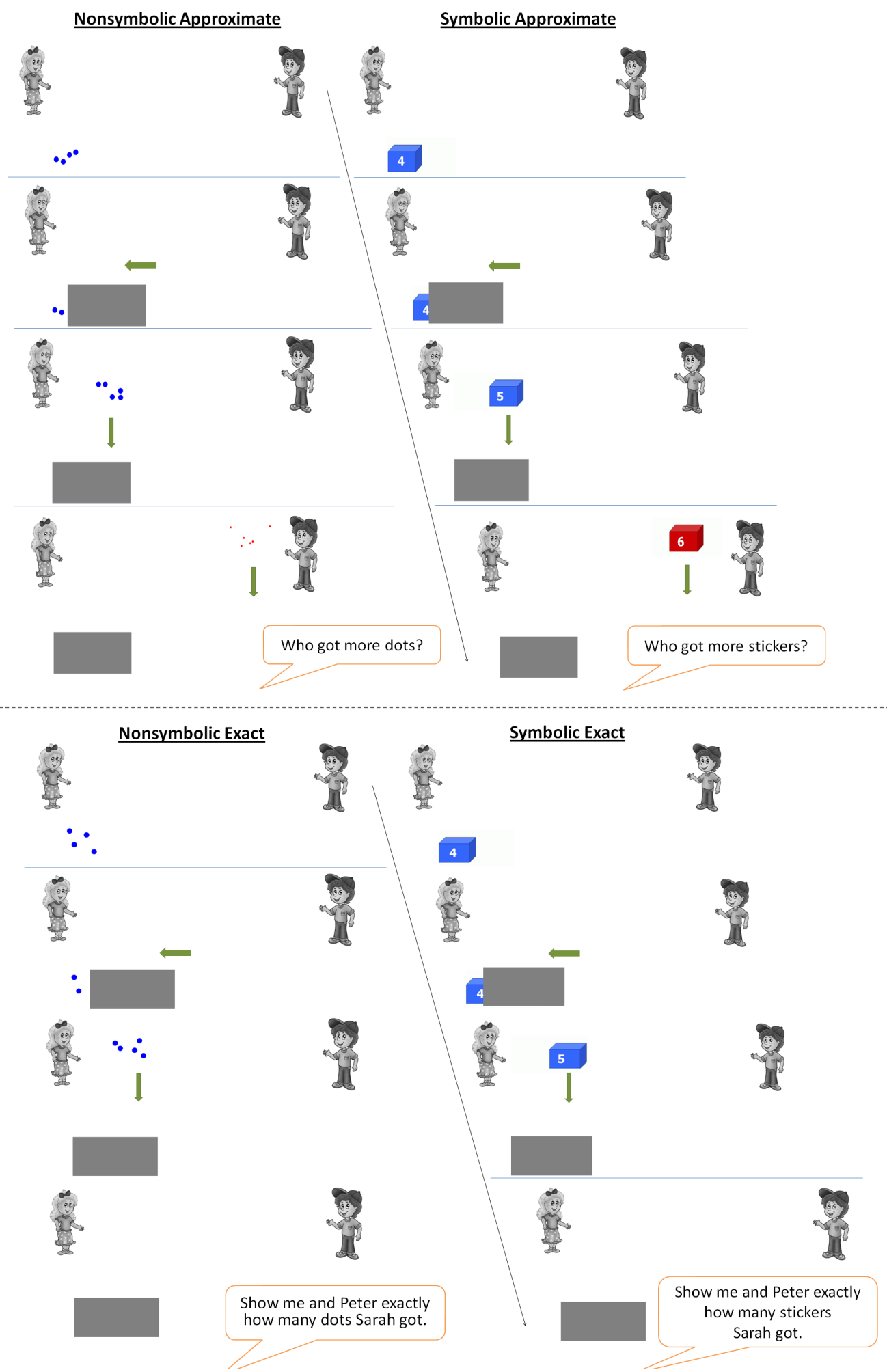

Figure 1. Example trials from the four addition problem formats. 
A) Nonsymbolic number line
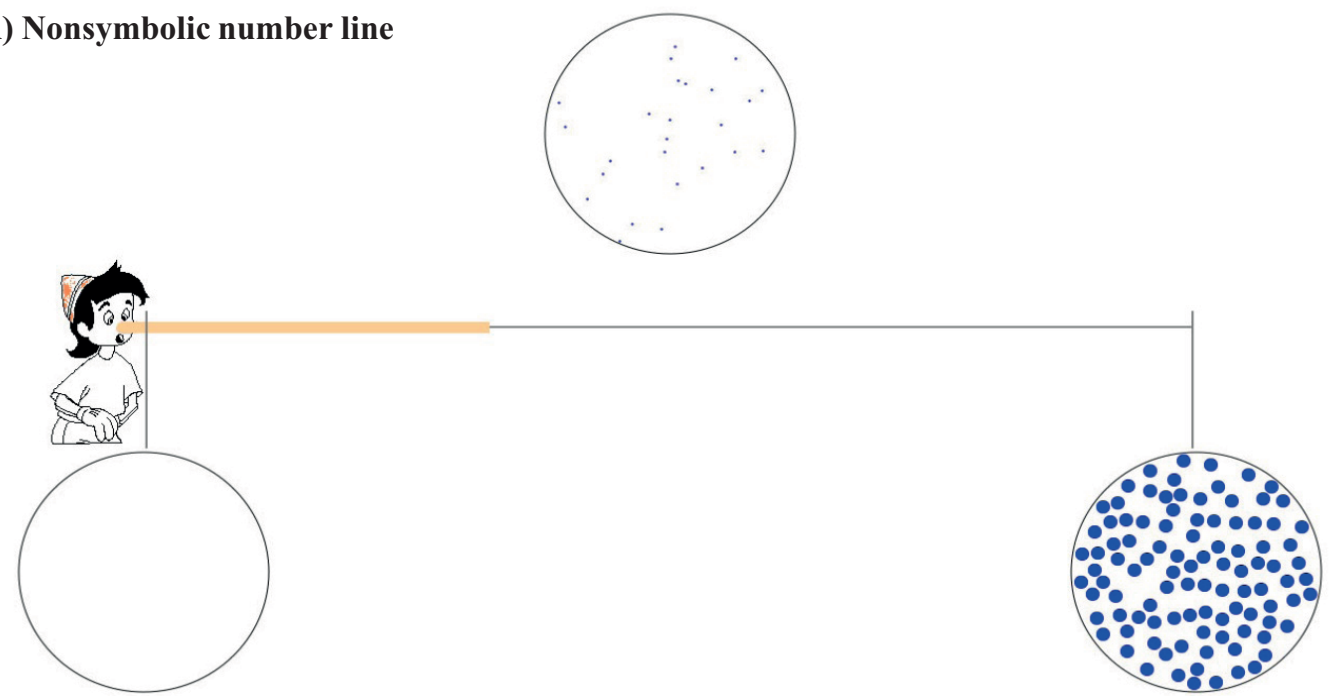

B) Symbolic number line

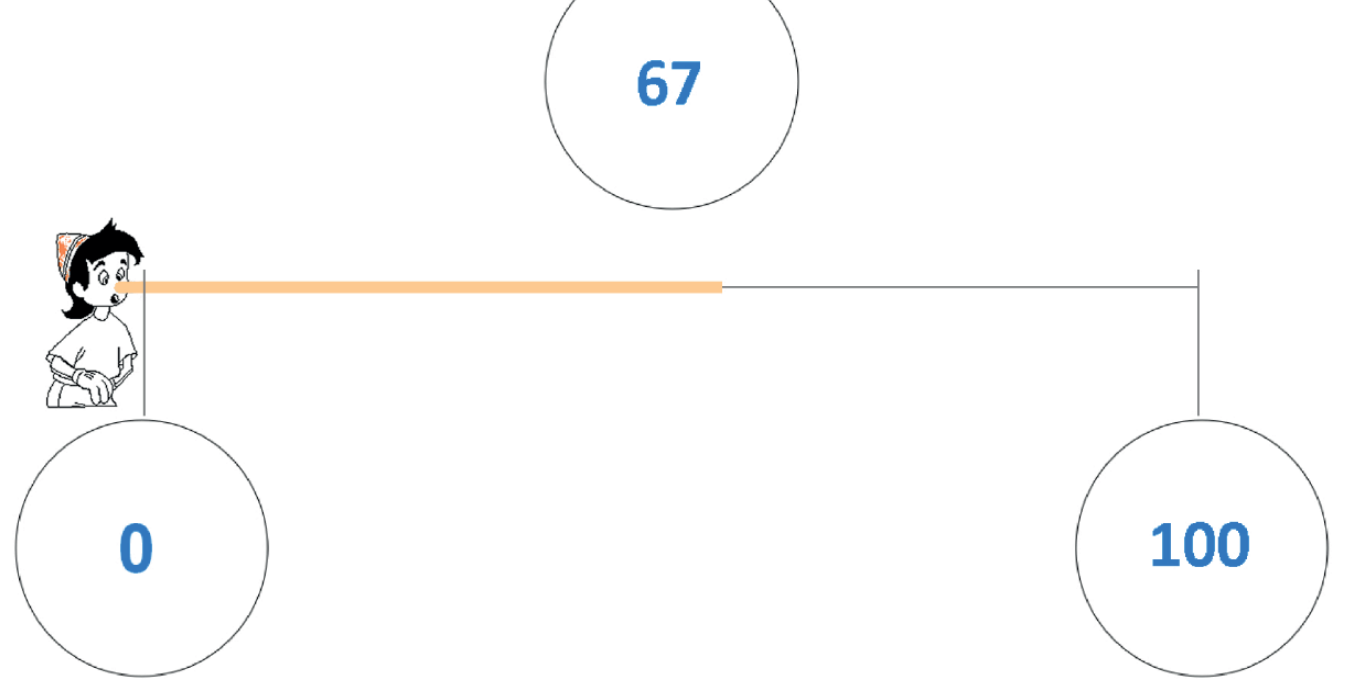

Figure 2. Example trials from the nonsymbolic and symbolic mental number line tasks. 


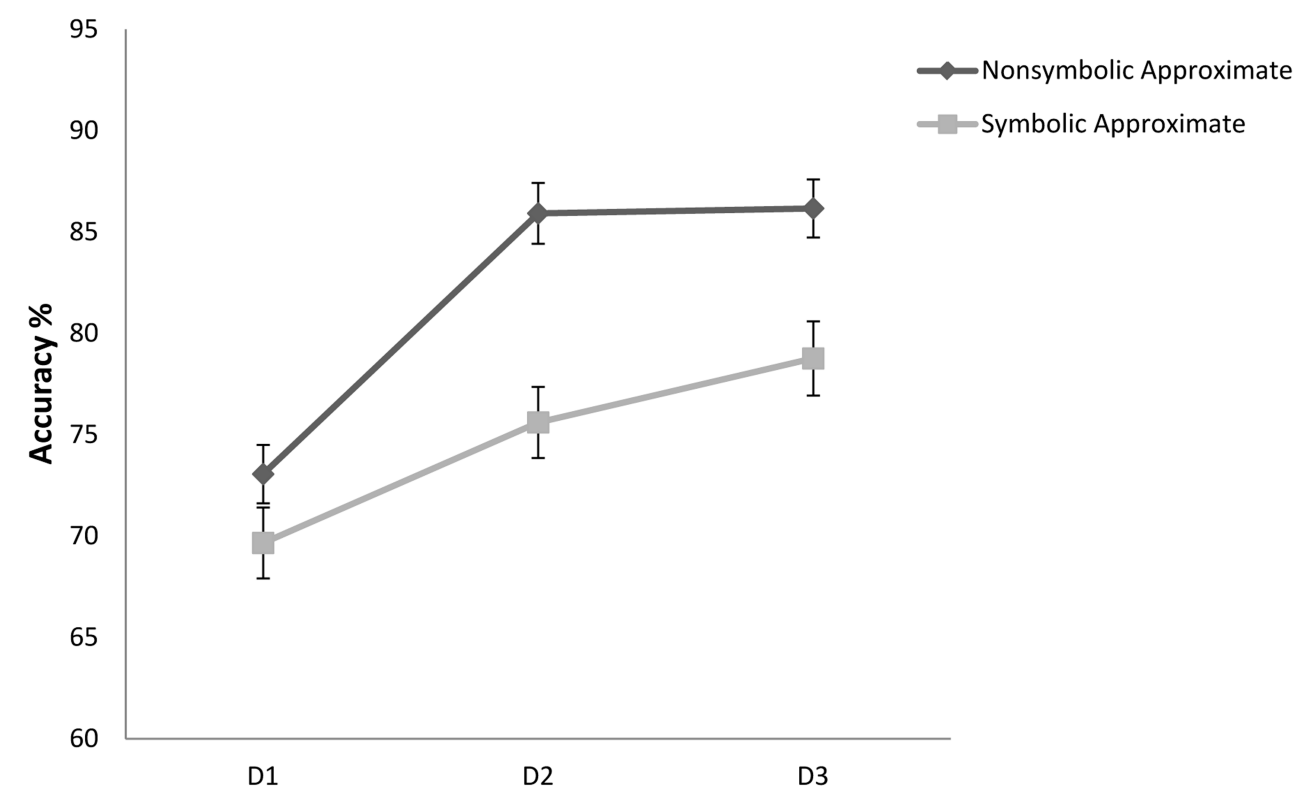

Figure 3. Distance effects in the two approximate addition tasks: nonsymbolic and symbolic.

The horizontal axis represents the three distance levels: from the small distance (D1), to the middle (D2) and largest one (D3). 
Table 1.

Hypothesized WM and Mental Number Line Estimation Predictors for Each of the Four Single-digit addition Problem Formats and the Expected Underlying Cognitive Mechanisms.

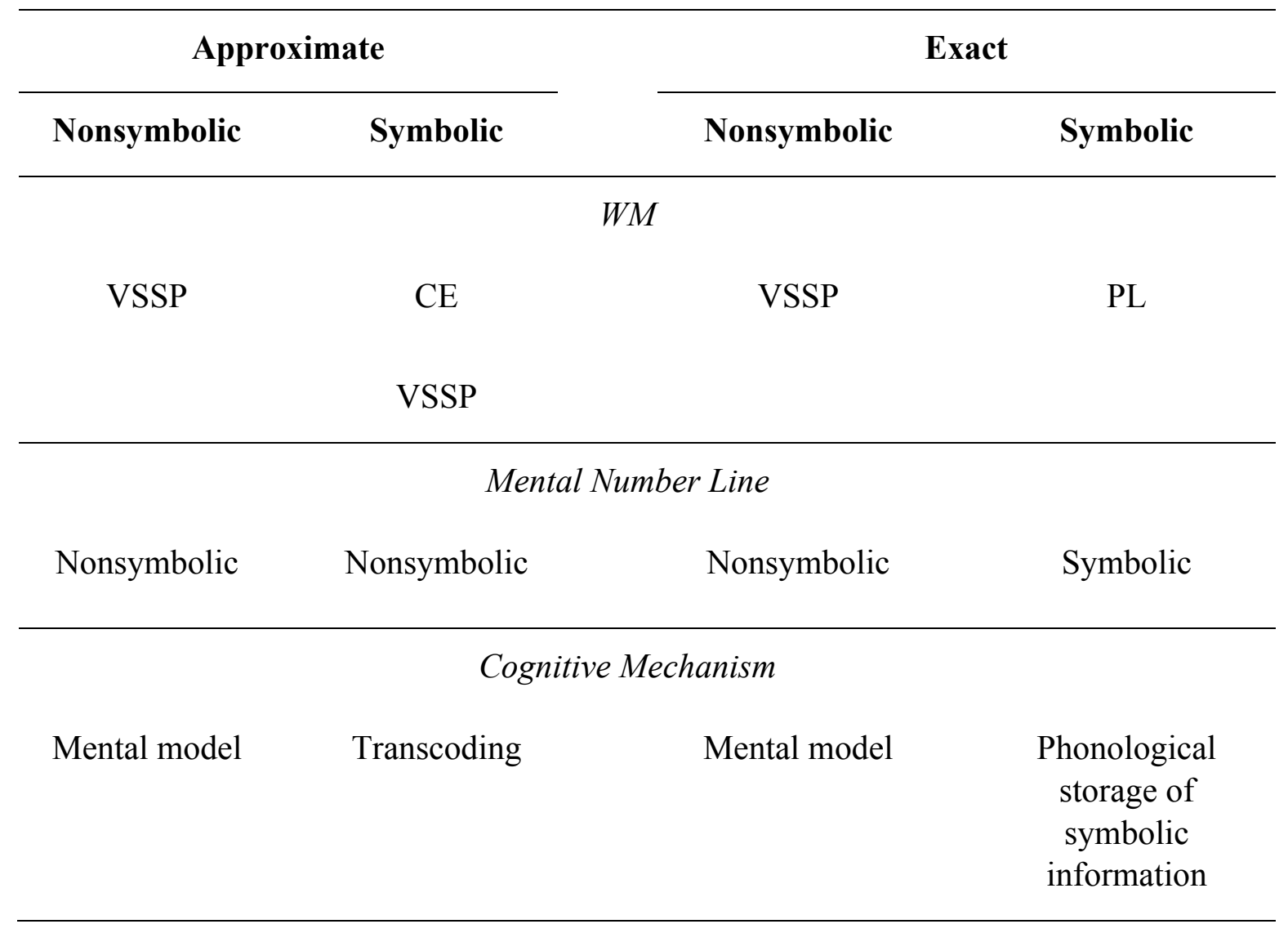


Table 2.

Descriptive Statistics.

\begin{tabular}{rcc}
\hline Addition measures & $M(S D)$ & Max \\
\hline Nonsymbolic Approximate & $19.61(2.63)$ & 24 \\
Symbolic Approximate & $17.92(3.60)$ & 24 \\
Nonsymbolic Exact & $13.54(4.60)$ & 24 \\
Symbolic Exact & $11.94(8.01)$ & 24 \\
\hline
\end{tabular}

\begin{tabular}{|c|c|c|}
\hline Predictors & & \\
\hline \multicolumn{3}{|l|}{ WM } \\
\hline Visuospatial (VSSP) & $14.31(5.32)$ & 30 \\
\hline Phonological Loop (PL) & $13.51(2.46)$ & 24 \\
\hline Central Executive (CE) & $4.59(2.10)$ & 24 \\
\hline \multicolumn{3}{|l|}{ Mental Number Line } \\
\hline Nonsymbolic NL PAE & $0.22(0.07)$ & 1 \\
\hline Symbolic NL PAE & $0.23(0.06)$ & 1 \\
\hline
\end{tabular}

Note. $\mathrm{PAE}=$ Percentage of Absolute Error, $M a x=$ Theoretical Maximum, NL PAE $=$ Number Line Percentage of Absolute Error. 
Table 3.

Correlations Between the Four Addition Problem Formats (1 -4) and Performance in the WM (5-7) and Mental Number Line (8-9) Tasks.

\begin{tabular}{|c|c|c|c|c|c|c|c|c|}
\hline Variables & 1 & 2 & 3 & 4 & 5 & 6 & 7 & 8 \\
\hline \multicolumn{9}{|c|}{ 1. Nonsymbolic Approximate } \\
\hline 2. Symbolic Approximate & $.27^{* *}$ & & & & & & & \\
\hline 3. Nonsymbolic Exact & $.34^{* * *}$ & $.39^{* * *}$ & & & & & & \\
\hline 4. Symbolic Exact & .13 & $.23^{*}$ & $.33^{* * *}$ & & & & & \\
\hline 5. VSSP & $.27^{* *}$ & $.35^{* * *}$ & $.51^{* * *}$ & $.25^{*}$ & & & & \\
\hline 6. PL & $.25^{*}$ & $.23^{*}$ & $.33^{* *}$ & $.32^{* * *}$ & $.36^{* * *}$ & & & \\
\hline 7. $\mathrm{CE}$ & .11 & $.36^{* * *}$ & $.39^{* * *}$ & .14 & $.32^{* * *}$ & $.26^{* *}$ & & \\
\hline 8. Nonsymbolic NL PAE & -.17 & $-.37^{* * *}$ & $-.39^{* * *}$ & $-.21^{*}$ & $-.27^{* *}$ & -.05 & $-.33^{* * *}$ & \\
\hline 9. Symbolic NL PAE & -.15 & $-.28^{* *}$ & $-.38^{* * *}$ & $-.36^{* * *}$ & -.16 & -.15 & $-.32^{* * *}$ & $.49^{* * *}$ \\
\hline
\end{tabular}

Note. VSSP $=$ Visuospatial, $\mathrm{PL}=$ Phonological Loop, $\mathrm{CE}=$ Central Executive, $\mathrm{NL}$ PAE $=$ Number Line Percentage of Absolute Error. $* p \leq .05, * * p \leq .01, * * * p \leq .001$. 
Table 4.

Stepwise Multiple Linear Regression Analyses for each of the four single-digit addition problem formats.

\begin{tabular}{|c|c|c|c|c|c|c|c|c|c|}
\hline Addition Problem format & Model & Predictors & $\boldsymbol{\beta}$ & Adjusted $R^{2}$ & $\Delta R^{2}$ & $F$ & $d f 2$ & $p$ & Note. \\
\hline Nonsymbolic Approximate & 1 & VSSP & .27 & .06 & .07 & 8.00 & 101 & .006 & Predictor's \\
\hline \multirow[t]{3}{*}{ Symbolic Approximate } & 1 & Nonsymbolic NL PAE & -.37 & .13 & .14 & 16.25 & 101 & .000 & standardized \\
\hline & 2 & VSSP & .27 & .19 & .07 & 8.47 & 100 & .004 & beta \\
\hline & 3 & $\mathrm{CE}$ & .21 & .22 & .04 & 4.75 & 99 & .032 & coefficient in \\
\hline \multirow[t]{3}{*}{ Nonsymbolic Exact } & 1 & VSSP & .51 & .25 & .26 & 34.68 & 101 & .000 & the \\
\hline & 2 & Symbolic NL PAE & -.31 & .33 & .09 & 13.94 & 100 & .001 & \\
\hline & 3 & $\mathrm{CE}$ & .18 & .35 & .03 & 4.00 & 99 & .049 & \\
\hline \multirow[t]{2}{*}{ Symbolic Exact } & 1 & Symbolic NL PAE & -.36 & .12 & .13 & 15.12 & 101 & .001 & (11000 () \\
\hline & 2 & PL & .27 & .19 & .07 & 8.83 & 100 & .004 & Adjusted $R^{2}$, \\
\hline
\end{tabular}

statistics for $\Delta R^{2}$ are reported. VSSP $=$ Visuospatial WM, PL $=$ Phonological Loop, $C E=$ Central Executive, NL PAE $=$ Number Line Percentage of Absolute Error. In all tests $d f 1=1$. 
Table 5.

Summarized Representation of Findings for the Four Single-digit Addition Problem Formats.

\begin{tabular}{|c|c|c|c|}
\hline \multicolumn{2}{|c|}{ Approximate } & \multicolumn{2}{|c|}{ Exact } \\
\hline Nonsymbolic & Symbolic & Nonsymbolic & Symbolic \\
\hline \multirow{3}{*}{ VSSP } & \multicolumn{2}{|c|}{ WM predictors } & \multirow{3}{*}{ PL } \\
\hline & VSSP & VSSP & \\
\hline & $\mathrm{CE}$ & $\mathrm{CE}$ & \\
\hline \multicolumn{4}{|c|}{ Mental Number Line Predictors } \\
\hline ns & Nonsymbolic & Symbolic & Symbolic \\
\hline \multicolumn{4}{|c|}{ Cognitive Mechanism } \\
\hline Mental model & Transcoding & Transcoding & $\begin{array}{l}\text { Phonological } \\
\text { storage of symbolic } \\
\text { information }\end{array}$ \\
\hline
\end{tabular}

Note. VSSP $=$ Visuospatial WM, PL $=$ Phonological Loop, $\mathrm{CE}=$ Central Executive, $\mathrm{ns}=$ no significant predictor. 
Table A1.

Testing Trials and Control Conditions in the Four Addition Problem Formats.

\begin{tabular}{|c|c|c|c|c|c|c|c|c|c|}
\hline \multirow[b]{2}{*}{ Trials } & \multirow{2}{*}{$\begin{array}{c}\text { First blue } \\
\text { array (B1) }\end{array}$} & \multirow{2}{*}{$\begin{array}{l}\text { Second blue } \\
\text { array (B2) }\end{array}$} & \multirow{2}{*}{$\begin{array}{c}\text { Correct Exact } \\
\text { Response }\end{array}$} & \multirow[b]{2}{*}{ Red array $(\mathbf{R})$} & \multirow[b]{2}{*}{ Distance } & \multirow{2}{*}{$\begin{array}{c}\text { Correct } \\
\text { Approximate } \\
\text { Response }\end{array}$} & \multicolumn{2}{|c|}{ Systematic Response Preferences ${ }^{a}$} & \multirow{2}{*}{$\begin{array}{l}\text { Continuous } \\
\text { quantity }\end{array}$} \\
\hline & & & & & & & Blue & Red & \\
\hline 1 & 2 & 6 & 8 & 7 & -1 & Sum blue & $1^{\mathrm{b}}$ & -1 & $\mathrm{~B}$ \\
\hline 2 & 3 & 1 & 4 & 3 & -1 & Sum blue & 1 & -1 & A \\
\hline 3 & 2 & 3 & 5 & 4 & -1 & Sum blue & 1 & -1 & $\mathrm{~B}$ \\
\hline 4 & 4 & 2 & 6 & 5 & -1 & Sum blue & 1 & -1 & A \\
\hline 5 & 1 & 1 & 2 & 3 & 1 & Red & -1 & 1 & $\mathrm{~B}$ \\
\hline 6 & 1 & 2 & 3 & 4 & 1 & Red & -1 & 1 & A \\
\hline 7 & 2 & 2 & 4 & 5 & 1 & Red & -1 & 1 & B \\
\hline 8 & 5 & 2 & 7 & 8 & 1 & Red & -1 & 1 & A \\
\hline 9 & 5 & 2 & 7 & 5 & -2 & Sum blue & 1 & -1 & B \\
\hline 10 & 2 & 3 & 5 & 3 & -2 & Sum blue & 1 & -1 & $\mathrm{~A}$ \\
\hline 11 & 5 & 3 & 8 & 6 & -2 & Sum blue & 1 & -1 & B \\
\hline 12 & 3 & 1 & 4 & 2 & -2 & Sum blue & 1 & -1 & A \\
\hline 13 & 2 & 4 & 6 & 8 & 2 & Red & -1 & 1 & B \\
\hline 14 & 1 & 2 & 3 & 5 & 2 & Red & -1 & 1 & A \\
\hline 15 & 1 & 1 & 2 & 4 & 2 & Red & -1 & 1 & B \\
\hline 16 & 2 & 2 & 4 & 6 & 2 & Red & -1 & 1 & A \\
\hline 17 & 5 & 2 & 7 & 4 & -3 & Sum blue & 1 & -1 & $\mathrm{~B}$ \\
\hline 18 & 1 & 4 & 5 & 2 & -3 & Sum blue & 1 & -1 & $\mathrm{~A}$ \\
\hline 19 & 3 & 3 & 6 & 3 & -3 & Sum blue & 1 & -1 & B \\
\hline 20 & 4 & 5 & 9 & 6 & -3 & Sum blue & 1 & -1 & A \\
\hline 21 & 1 & 1 & 2 & 5 & 3 & Red & -1 & 1 & B \\
\hline 22 & 2 & 1 & 3 & 6 & 3 & Red & -1 & 1 & A \\
\hline 23 & 2 & 2 & 4 & 7 & 3 & Red & -1 & 1 & B \\
\hline 24 & 2 & 1 & 3 & 6 & 3 & Red & -1 & 1 & $\mathrm{~A}$ \\
\hline
\end{tabular}

a These columns present the control conditions for the case that children tended to press only the "Blue" button, or only the "Red" button

$1=$ if this response preference is chosen the correct answer is predicted. $-1=$ if chosen, does not predict correct answer for this trial.

Control conditions for the physical features of the dots: A =Dot size, total dot surface area, total dot contour length and density were positively correlated with numerosity whereas array size was negatively correlated with numerosity. $\mathrm{B}=$ opposite relations. 
Table A2.

Examination of Systematic Response Preferences and Continuous Quantity Effects

\begin{tabular}{ccccc}
\hline $\begin{array}{c}\text { Control } \\
\text { Condition }\end{array}$ & $\begin{array}{c}\text { Number of } \\
\text { Trials }\end{array}$ & $\begin{array}{c}\text { Mean } \\
\text { Accuracy \% }\end{array}$ & $\boldsymbol{p}$ value & $\begin{array}{c}\text { > chance } \\
\mathbf{( 5 0 \% )}\end{array}$ \\
\hline \multicolumn{5}{c}{ Nonsymbolic } \\
Approximate Addition \\
Blue & 12 & 83.02 & .000 & yes \\
Red & 12 & 80.71 & .000 & yes \\
A & 12 & 80.79 & .000 & yes \\
B & 12 & 82.95 & .000 & yes \\
\hline \multicolumn{5}{c}{ Symbolic Approximate Addition } \\
Blue & 12 & 72.53 & .000 & yes \\
Red & 12 & 76.62 & .000 & yes \\
\hline
\end{tabular}

